\title{
Phase Contrast X-Ray Synchrotron Imaging: Opening Access to Fossil Inclusions in Opaque Amber
}

\author{
Malvina Lak, ${ }^{1,2}$ Didier Néraudeau, ${ }^{2}$ André Nel, ${ }^{3}$ Peter Cloetens, ${ }^{1}$ Vincent Perrichot, ${ }^{4}$ \\ and Paul Tafforeau ${ }^{1,5, *}$ \\ ${ }^{1}$ European Synchrotron Radiation Facility, BP 220, F-38043 Grenoble Cedex, France \\ ${ }^{2}$ Géosciences Rennes, UMR 6118, CS 74205, 35042 Rennes Cedex, France \\ ${ }^{3}$ CNRS UMR 5202, Muséum National d'Histoire Naturelle, Entomologie, CP 50, F-75005 Paris, France \\ ${ }^{4}$ Museum für Naturkunde der Humboldt-Universität zu Berlin, D-10115 Berlin, Germany \\ ${ }^{5}$ LGBPH Poitiers, UMR 6046, F-86022 POITIERS Cedex, France
}

\begin{abstract}
A significant portion of Mesozoic amber is fully opaque. Biological inclusions in such amber are invisible even after polishing, leading to potential bias in paleoecological and phylogenetic studies. Until now, studies using conventional X-ray microtomography focused on translucent or semi-opaque amber. In these cases, organisms of interest were visualized prior to X-ray analyses. It was recently demonstrated that propagation phase contrast X-ray synchrotron imaging techniques are powerful tools to access invisible inclusions in fully opaque amber. Here we describe an optimized synchrotron microradiographic protocol that allowed us to investigate efficiently and rapidly large amounts of opaque amber pieces from Charentes (southwestern France). Amber pieces were imaged with microradiography after immersion in water, which optimizes the visibility of inclusions. Determination is not accurate enough to allow precise phylogenetic studies, but provides preliminary data on biodiversity and ecotypes distribution; phase contrast microtomography remains necessary for precise determination. Because the organisms are generally much smaller than the amber pieces, we optimized local microtomography by using a continuous acquisition mode (sample moving during projection integration). As tomographic investigation of all inclusions is not practical, we suggest the use of a synchrotron for a microradiographic survey of opaque amber, coupled with microtomographic investigations of the most valuable organisms.
\end{abstract}

Key words: opaque amber, synchrotron imaging, propagation phase contrast, microradiography, microtomography, fossil arthropods, continuous acquisition mode

\section{INTRODUCTION}

During the last few years, several studies have been performed on fossil biological inclusions preserved in "opaque" and semi-opaque amber (fossil tree resin) using conventional X-ray computed tomography (CT) and more recently microtomography ( $\mu \mathrm{CT}$ ) (Grimaldi et al., 2000; Polcyn et al., 2003; Cnudde et al., 2006; Henderickx et al., 2006; Dierick et al., 2007). However, in these cases, the ambers studied were not truly opaque, because the inclusions were roughly recognized either with the naked eye or under a microscope before X-ray imaging experiments. However some types of truly opaque amber exist, particularly among the Mid-Cretaceous ambers, like the ones from Charentes, France (Perrichot, 2004, 2005; Tafforeau et al., 2006). In such cases, it is impossible to see inside the amber pieces to

Received October 9, 2007; Accepted October 24, 2007

*Corresponding author. E-mail: paul.tafforeau@esrf.fr investigate possible inclusions. Although conventional $\mu \mathrm{CT}$ could be used to image inclusions in such kind of amber, it is in practice nearly impossible without prior knowledge of where the inclusions are located and without rough characterization (basic taxonomical determination and size measurements). These technical limitations explain why fossil organisms in opaque amber are never studied, leading to potential paleoecological characterization bias regarding to the amount of opaque amber in some sites (more than $80 \%$ in Charentes for example).

Tafforeau et al. (2006) demonstrated that propagation phase contrast synchrotron imaging (Snigirev et al., 1995; Cloetens et al., 1996a) is an effective way to reveal and precisely locate inclusions in fully opaque amber prior to microtomographic investigations. When using a partially coherent X-ray beam such as the one produced by a thirdgeneration synchrotron, increasing the distance between the sample and the detector allows efficient edge detection contrast (i.e., propagation phase contrast effect) superimposed on absorption one. That technique gives good 
results for isolated amber pieces, but is not an optimal way to survey a large number of amber samples due to their various sizes and shapes.

Phase contrast imaging was originally developed using synchrotron sources, but the effect can also be observed in some cases using microfocus X-ray tubes (Wilkins et al., 1996), and was used recently to image small inclusions in amber using $\mu \mathrm{CT}$ (Dierick et al., 2007). However, it is nearly impossible to use conventional X-ray sources to search for invisible inclusions in large opaque amber samples because coherence properties of such sources are significantly lower than what can be obtained using synchrotron sources. Coherence properties depend on the source size, on the distance between the source and the sample, and on the energy of the beam (Snigirev et al., 1995; Cloetens et al., 1996a; Baruchel et al., 2001). In the case of conventional sources, even if the source size can be rather small (e.g., $0.9 \mu \mathrm{m}$ according to Dierick et al., 2007), the relatively short distance between source and detector requires low energy and high resolution to obtain useful phase contrast effects. Such conditions are not compatible with a large-scale survey of amber pieces because they do not allow for imaging many samples or large samples.

In the present article, we demonstrate an optimized imaging protocol, using propagation phase contrast X-ray synchrotron microradiography to efficiently survey large amounts of opaque amber after immersion in water, followed by phase contrast microtomography for precise characterization of selected organisms.

\section{Materials and Methods}

The amber samples come from four localities of the Charentes region in southwestern France. Two amber deposits, from Archingeay-Les Nouillers and Cadeuil, are Late Albian in age (Néraudeau et al., 2002, in press) whereas Champniers and Fouras are dated as Early Cenomanian (Néraudeau et al., 2003; Perrichot et al., 2007). Samples range from 1 to $200 \mathrm{~cm}^{3}$, representing approximately 640 amber pieces for a total weight of $2 \mathrm{~kg}$.

Tafforeau et al. (2006) demonstrated the possibilities of phase contrast synchrotron imaging for investigation of isolated opaque amber samples, yet was unable to apply this technique to large numbers and sizes of amber pieces. To improve upon this initial approach, we developed a specific microradiographic protocol involving water submersion and standardized sample holders to image several pieces of various sizes and shapes simultaneously with optimum contrast.

\section{Phase Imaging and Water Immersion Effect}

Absorption of a monochromatic X-ray beam by a sample is related to sample thickness through the Beer-Lambert relation $I=I_{0} e^{-\mu d}$ where $I$ is the transmitted beam, $I_{0}$ the incoming beam, $\mu$ the linear absorption coefficient (depending on the density and to a lesser extent on the chemical composition of the material), and $d$ the thickness of the sample. When using propagation phase contrast imaging, absorption and phase information superimpose on the same radiograph. In the case of homogeneous samples of different shapes and thicknesses (e.g., amber pieces), the absorption contribution to the radiographic contrast can be important, potentially impeding optimal phase contrast for the entire sample. Tafforeau et al. (2006) showed that fossil inclusions in amber are nearly invisible with absorption contrast whereas they are well visualized when using phase contrast. The inclusion detection in opaque amber is optimized by increasing the proportion of propagation phase contrast to absorption contrast, leading to enhanced visibility of each inclusion relative to the visibility of the entire amber piece.

Because amber and water densities are similar, they should exhibit comparable X-ray absorption and phase properties. Submerging the amber samples under water in rectangular vats is expected to lead to a nearly constant $\mathrm{X}$-ray absorption of the whole volume regardless of the thickness and shape of amber pieces. Phase contrast effects are encountered at boundaries between mediums with different densities. In amber, these effects are observed not only for fossil biological inclusions, but also on cracks, bubbles, and at the outer surface of amber pieces. Immersion should therefore reduce the unwanted phase contrast effect caused by the outer surface and cracks reaching the surface, without affecting the visibility of the inclusions inside. Comparable immersion technique was previously used for phase matching using X-ray interferometers (Momose, 1995; Beckmann et al., 1997).

\section{Sample Preparation Protocol for Microradiography}

Amber pieces were fixed using thermoplastic glue on 5-mmthick Plexiglas plates of $50 \mathrm{~mm} \times 100 \mathrm{~mm}$. Thermoplastic glue was used for its good physical and water resistance properties, its density (close to that of water), its fast solidification, and because it can be removed by moderate heating (no visible effect on the amber) without using solvents that might affect amber samples. The plate size was selected in order to fit within the travel range of the different motors and to yield a reasonable data size per plate. Each plate was inserted in a Plexiglas rectangular vat allowing complete immersion in water (Fig. 1), the fixation being insured by putting the lower part of the plate in a longitudinal $1-\mathrm{cm}$ deep slot. We used demineralized water in order to limit potential chemical reactions with amber. We boiled it to avoid bubbles due to the dissolved gas. Depending on the maximum thickness of amber samples glued on the plates, we used vats of 40 and $60 \mathrm{~mm}$ depth. In both cases, the vat height was $90 \mathrm{~mm}$ and its width $140 \mathrm{~mm}$ (leaving $20 \mathrm{~mm}$ for beam reference pictures through water only on each side of the plate). 


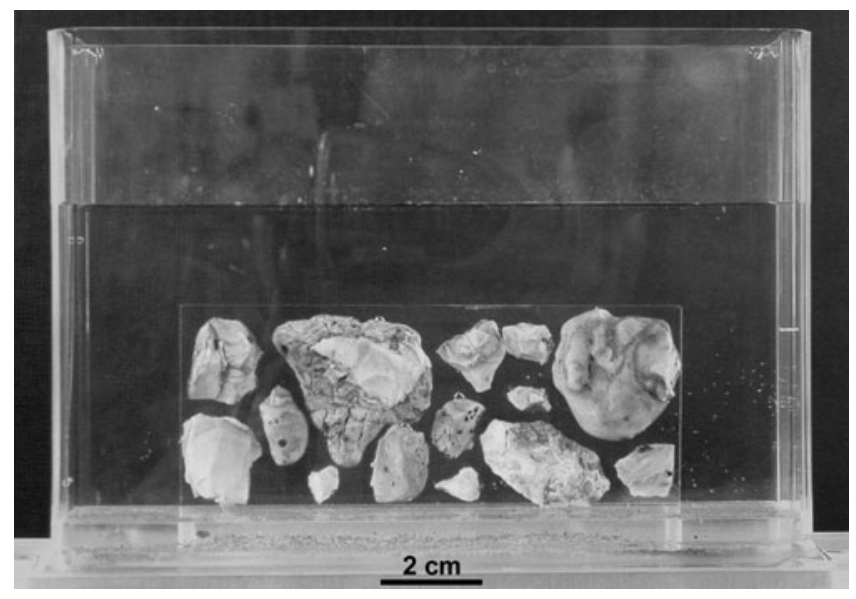

Figure 1. Sample holder developed for phase contrast microradiography of opaque amber. Amber pieces are glued on a Plexiglas plate fixed vertically in a rectangular Plexiglas vat filled with demineralized boiled water used to normalize X-ray absorption and to optimize phase contrast visibility of inclusions.

\section{Microradiographic Acquisition Parameters}

All the microradiographic experiments were performed at the European Synchrotron Radiation Facility (ESRF, Grenoble, France), on the long imaging beamline ID19 (145 m between the source and the sample). We used a monochromatic beam set at an energy of $35 \mathrm{keV}$ with a double crystal silicon 111 monochromator. To obtain the propagation phase contrast effect, the propagation distance (i.e., the distance between the sample and the detector) was set to $990 \mathrm{~mm}$. The detector was composed of a FReLoN (Fast Read-out Low Noise) charge coupled device (CCD) camera with $2048 \times 2048$ pixels, mounted on an optical lens-based magnification system coupled to a 5 - $\mu \mathrm{m}$-thick Gadox $\left(\mathrm{Gd}_{2} \mathrm{O}_{2} \mathrm{~S}: \mathrm{Tb}\right)$ scintillator screen. That system yields an isotropic pixel size of $5.06 \mu \mathrm{m}$, but the effective pixel size at the sample level was $5.03 \mu \mathrm{m}$ due to the sample/detector distance and to the slight beam divergence. The synchrotron storage ring filling mode was 16 bunches (90 mA current) and we used a $32-\mathrm{mm}$ period undulator insertion device to produce the X-ray beam. To obtain pictures with a high dynamic range, we used an integration time of $2 \mathrm{~s}$ per projection. At $35 \mathrm{keV}$, the vertical size of the beam produced by the undulator was smaller than the maximum detector field of view; the resulting field of view of each radiograph was then limited to $5.79 \mathrm{~mm}(\mathrm{v}) \times 10.30 \mathrm{~mm}(\mathrm{~h})$, corresponding to $1152 \times 2048$ pixels. To cover the whole surface of the plate, we performed a mosaic radiograph using an automatic acquisition protocol to acquire subparts, reference images (two pictures per line, one on each side of the plate in water only), and dark noise images (10 pictures at the end of the scan). The lateral distance between two projections on the same line was $8 \mathrm{~mm}$ and the vertical one between two consecutive lines was $4 \mathrm{~mm}$. To image the whole surface of a single plate, we then acquired 160 projections (including the references) in 10 rows and 16 columns.

\section{Processing of Microradiographic Data}

Each projection was divided by the beam reference (weighted average between the two references of the corresponding line according to the lateral position of the projection) after dark noise subtraction in both the picture and its reference.

Mosaics were collated using an automatic script developed with MATLAB Release 2006a (The MathWorks Inc., Natick, MA). The final 32-bit images size was $720 \mathrm{Mb}$. They were converted into 8-bit images after determination of contrast values (optimizing inclusion visibility), yielding a fourfold data reduction and a final size of each mosaic radiograph of $180 \mathrm{Mb}$. Each mosaic microradiograph was then examined with Photoshop 7.0 (Adobe Systems Inc., San Jose, CA). All spotted biological inclusions were located, roughly determined, and measured in order to obtain an overall characterization of the diversity of preserved organisms, followed by selection of the most valuable ones. This includes organisms that are not preserved in translucent amber, have a poor or unknown fossil record, or provide access to structures that are not available on other individuals. These organisms are thus of great paleontological, taxonomic, or phylogenetic value for improving our knowledge of the origin and evolutionary history of organisms. For each of them the best parameters must be determined to proceed with $\mu \mathrm{CT}$.

\section{Propagation Phase Contrast X-ray Synchrotron Microtomography}

Some of the selected biological inclusions were then imaged in three dimensions (3D) using propagation phase contrast $\mathrm{X}$-ray synchrotron $\mu \mathrm{CT}$ on the beamlines ID19 and BM05 at the ESRF. Amber pieces displaying valuable inclusions were unglued from the Plexiglas plates and were fixed on sample holders using honey wax. We did not use immersion in water for $\mu \mathrm{CT}$. Depending on the sample size, we used isotropic voxel sizes ranging from 0.7 to $16 \mu \mathrm{m}$, the detector system being basically the same as for microradiography (scintillator screen, lens magnification system, CCD FreLoN camera). To image various kinds of samples, we adapted the propagation distance to obtain a moderate phase contrast effect (optimal distance for phase contrast in $\mu \mathrm{CT}$ on opaque amber is roughly half of the distance used for microradiography). These distances ranged from $100 \mathrm{~mm}$ at $0.7 \mu \mathrm{m}$ voxel size to $990 \mathrm{~mm}$ at $16 \mu \mathrm{m}$ voxel size. In the latter case the distance was set to the maximum allowed by the instrument. Using phase contrast for microtomography experiments increases the visibility of small structures undetectable in absorption mode (Tafforeau et al., 2006; Die- 

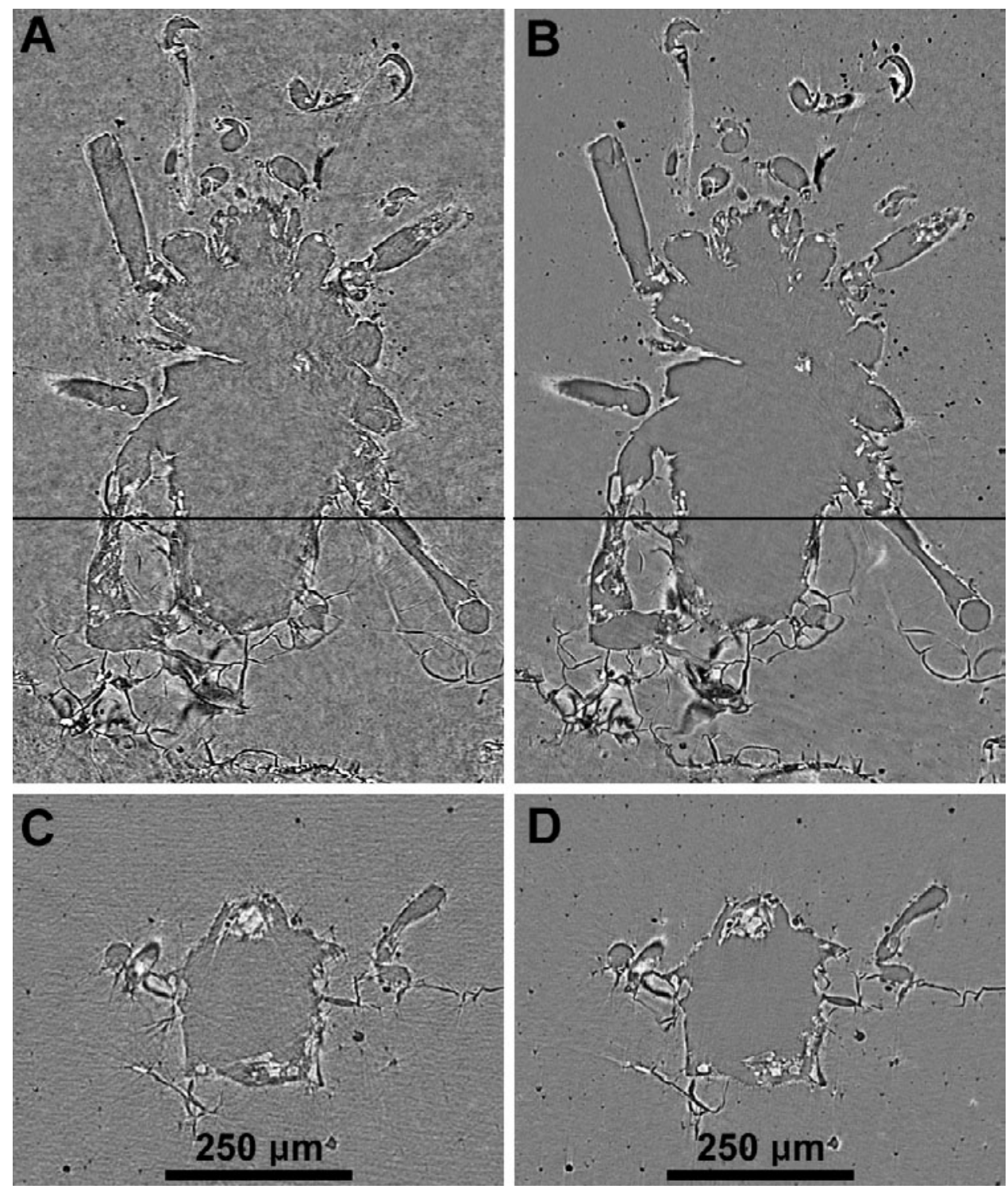

Figure 2. Comparison between step-by-step and continuous scan acquisition modes in local tomography, on a 0.8-mm fossil acarian preserved in opaque amber. The isotropic voxel size was $1.4 \mu \mathrm{m}$ in both cases, corresponding to a reconstruction field of view of $1.4 \mathrm{~mm}$ wide. The whole amber piece measuring $17 \times 10 \mathrm{~mm}$ at the inclusion level, the scans were performed with only $1.15 \%$ of the sample in the field of view. A,C: Microtomographic vertical and axial slices (the horizontal black line representing the position of the axial slice) obtained in step-by-step scan mode using 1500 projections over $180^{\circ}$. Structures out of the field of view generate strong noise on the reconstructed data. B,D: Same slices obtained in continuous scan mode using only 1200 projections over $180^{\circ}$. Continuous acquisition mode significantly decreases the noise due to structures out of the field of view, because they are blurred by the rotation movement during projection integration time. Sample orientation being different between the two scans, data sets were aligned using 3D registration of the two volumes.

rick et al., 2007). In some cases, propagation phase contrast can reveal structures that are smaller than the pixel size (Cloetens et al., 1996b). It is particularly important to image bristles or insects wings because these structures have special importance for taxonomic assessment but are generally extremely thin relative to the size of the whole organism.
Depending on the size of the amber pieces, the energy of the monochromatic beam was tuned from 20 to $35 \mathrm{keV}$; larger blocks required higher energies. In most cases, the selected organisms were much smaller than the amber pieces (Fig. 2). We necessarily used local tomography with a field of view significantly smaller than the lateral dimensions of 
the sample. In addition to an adapted reconstruction algorithm (filtered backprojection with adapted padding of the data, implemented in the ESRF software PyHST), we used a specific acquisition protocol based on a continuous scan. In opposition to step by step acquisition where image integration and motor movement are done sequentially, during a continuous scan, the sample rotates without stopping at a constant speed, projections being taken "on the fly" during the movement. Each projection then corresponds to an angular integration. This technique tends to blur structures on radiographs that are far from the center of rotation (increasing with the distance from the center of rotation), without modifying significantly the ones in the field of view, provided that the angular step is small enough to ensure that structures at the border of the field of view do not move by more than one pixel during a single projection. On the reconstructions, this approach yields spectacular results, as it reduces the high frequency noise due to structures outside the field of view (Fig. 2), facilitating efficient 3D processing of the data. That technique is sometimes used in conventional microtomographs to increase the quality of local tomography. The number of projections was selected in order to obtain high quality results on the whole field of view using a continuous scan over $180^{\circ}$ (as synchrotron $\mu \mathrm{CT}$ is characterized by parallel beam geometry). For a lateral field of view of 2048 pixels, we used typically 1500 projections per scan, corresponding to an angular step of $0.12^{\circ}$.

\section{Results}

\section{Observed Water Immersion Effect}

Without water, thick amber pieces can appear very dark on radiographs in their thickest parts, limiting inclusion detection in areas with different thickness (Fig. 3A). When immersed in water, the resulting absorption images (amber + water) appear quite homogeneous. This allows determination of optimal contrast values for visualization of inclusions that can be applied to the whole mosaic radiograph, rather than for a specific thickness of amber.

Selectively reduced phase contrast effects are also observed as expected. Outlines of the amber pieces are almost invisible whereas overall inclusion visibility is increased (Fig. 3B), leading to easier detection and characterization. Most of the bloc surface structures are invisible, and water penetration into cracks reaching the surface renders most of them almost invisible on microradiographs (Fig. 3C,D).

\section{Detection and Characterization of Biological Inclusions}

In $48 \mathrm{~h}$ of beam time on the beamline ID19, we fully imaged 91 plates, representing a total surface of amber of $3640 \mathrm{~cm}^{2}$. Initial data analysis took about $50 \mathrm{~h}$, including

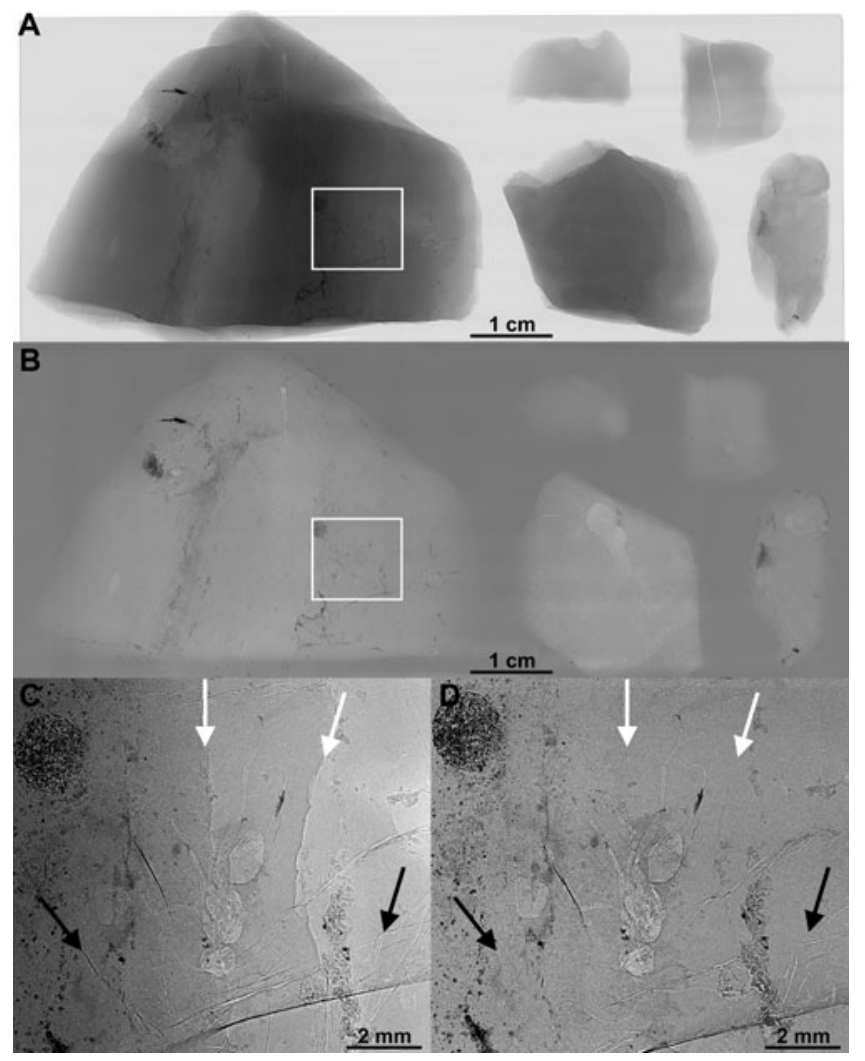

Figure 3. A: Mosaic microradiograph of a plate with amber pieces imaged in air. B: Microradiograph of the same plate imaged after immersion in demineralized boiled water. Water normalizes absorption on the imaged area leading to homogeneous average gray level and increased visibility of inclusions. One of the amber pieces was removed and glued back in a different position and the overall plate angle was slightly different between the two experiments. C,D: Details of A and B, respectively, showing the effect of water on surface structures (white arrows) and on cracks in amber (black arrows). Water completely removes surface structures and low frequency gray level variations due to different thicknesses of amber and greatly reduces the visibility of cracks by filling them when they reach the outer surface. It yields a better overall visibility of the inclusions and of their fine structures.

automatic processing time to collate the original projections. After that first survey, 356 fossil organisms were definitively recognized as nearly complete animal inclusions and tentatively identified (up to the ordinal level in most of the cases and up to the family level in exceptional cases). Such determination was possible for 189 inclusions, corresponding to $53 \%$ of the organisms discovered. Many isolated parts of animals were also recognized, as well as several plant remains.

Mosaic radiographs were used to locate precisely the inclusions in the amber pieces. Once the sample was aligned in two dimensions with radiographs, it was easy to align the third dimension directly on the microtomograph by 

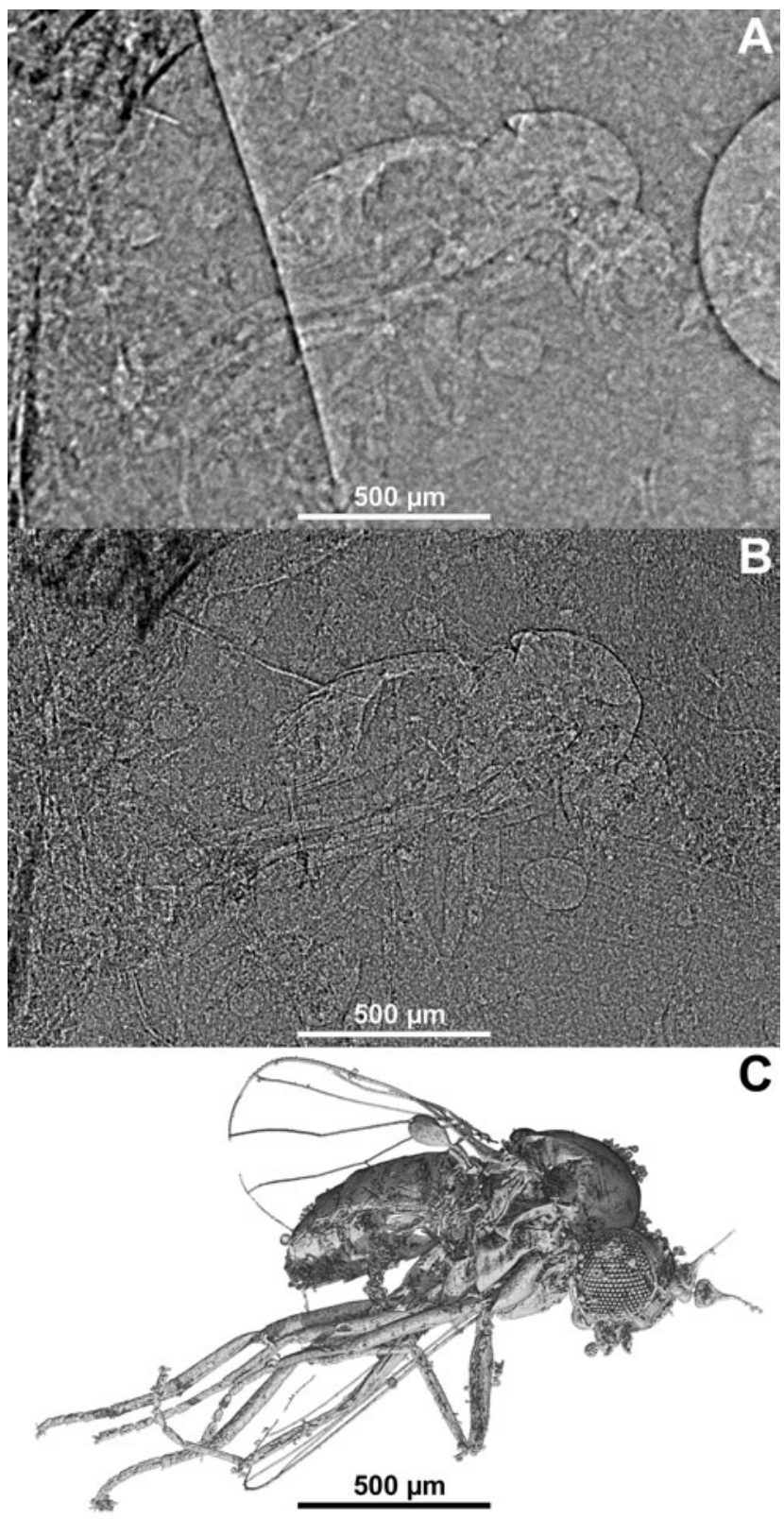

Figure 4. A: Phase contrast microradiograph of a fossil Diptera obtained with a 5.03- $\mu \mathrm{m}$ pixel size using a double Si 111 crystal monochromator. B: Same fossil imaged with a $1.4-\mu \mathrm{m}$ pixel size with a multilayer monochromator. Smaller details are visible but the overall inclusion visibility is reduced due to the lower coherence, leading to reduced visualization of the complete animal. C: Phase contrast microtomography with an isotropic $1.4-\mu \mathrm{m}$ voxel size. Far more details can be observed on $3 \mathrm{D}$ virtual extraction of that Diptera, allowing its assignment to the genus Microphorites Hennig (1971) that would not have been possible on radiographs.

following the displacement of the organism during the rotation. After tomographic reconstruction and 3D segmentation (i.e., virtual extraction of the biological inclusion from the surrounding resin), it was possible to determine
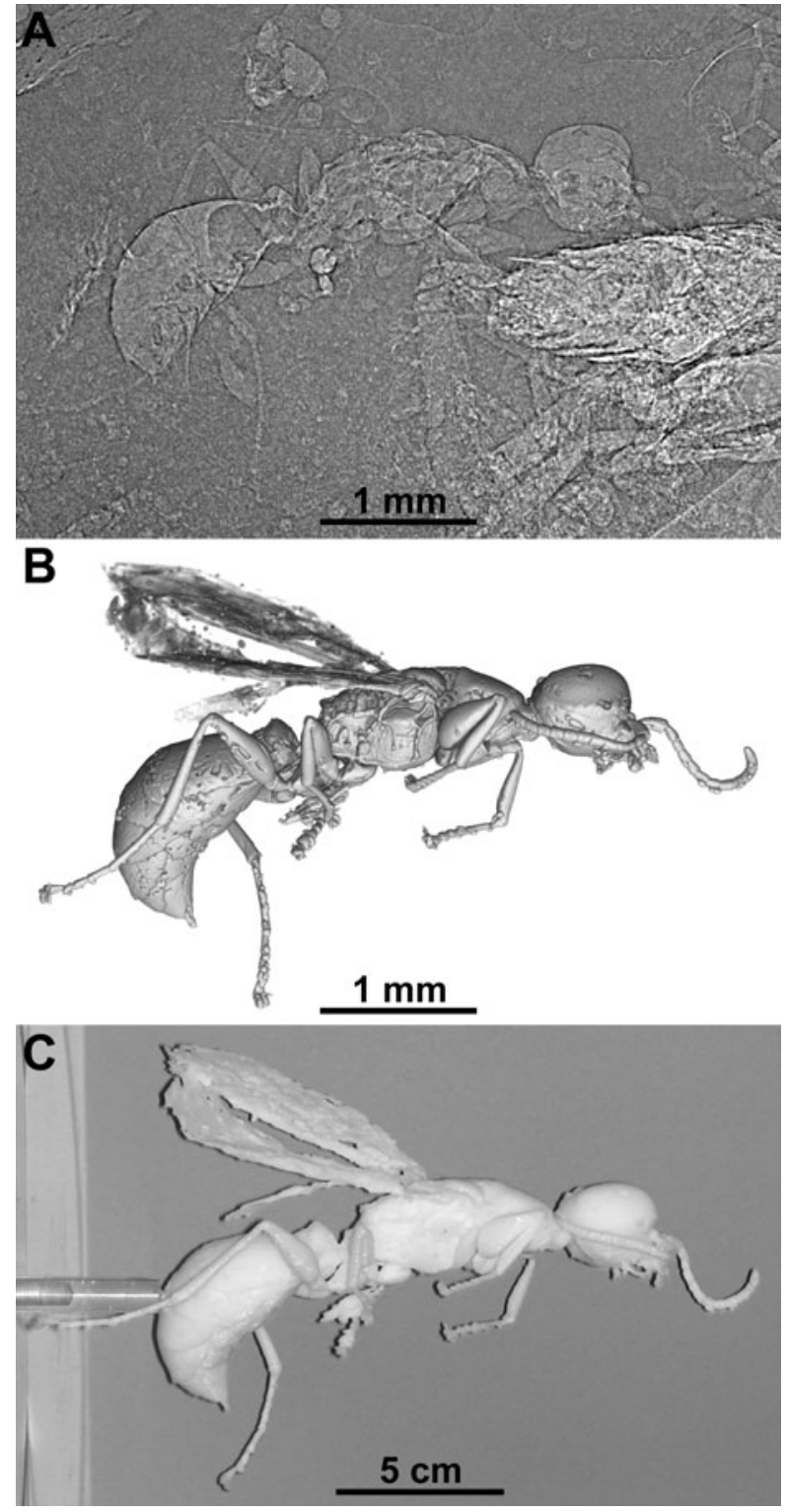

Figure 5. A: Phase contrast microradiography of a fossil Hymenoptera obtained with a $5.03-\mu \mathrm{m}$ pixel size. Part of another animal (a Blattaria) can be seen on the right lower corner of the picture. B: Result of 3D segmentation on a phase contrast microtomographic data set with an isotropic voxel size of $5.03 \mu \mathrm{m}$. Many more details are visible on the 3D data than on the microradiograph, allowing its assignment to the wasp family Falciformicidae (the accurate description will be provided elsewhere). Wings that were poorly visible on the radiograph were successfully extracted from 3D data due to the moderate phase contrast used for tomography. C: 3D printout of the same wasp that will be used as part of the holotype once the new species is fully described.

all the scanned animals up to the generic or to the specific level (Figs. 4, 5). In most cases, $\mu \mathrm{CT}$ confirmed the validity of the radiographic taxonomic determination at the order level. 


\section{DiscusSION}

\section{Interest of the Use of Water Immersion}

Absorption normalization of mosaic radiographs with water immersion facilitates standardization of data acquisition and processing, as well as optimization of the detection and determination level. By using this protocol, the size and shape of amber samples are no longer limitations, leading to more effective and efficient opaque amber investigation.

Despite the fact that water has no effect on amber itself, we noticed a substantial modification (and sometimes fragmentation) of some amber pieces after imaging, as demonstrated by white surface depositions and a sulphur smell. Given that observation of the corresponding radiographs revealed dense absorbing structures, we propose that this effect could be due to chemical reactions between water and pyrite $\left(\mathrm{FeS}_{2}\right)$, these iron sulphur crystals being commonly observed in ambers. Using another solvent could minimize this effect; nevertheless, such solvent would have to exhibit a density close to amber, be harmless for the resin and for the Plexiglas vat, and be fluid enough to fill the fractures. Given the difficulties of finding such a solvent, and the fact that only a very small proportion of the amber pieces had serious troubles due to immersion, water can be considered as a very effective medium for this kind of imaging, even if it is not ideal.

\section{Efficiency of Detection and Recognition of Biological Inclusions}

The protocol detailed here appears to be extremely effective for quantitative detection, with 356 animal inclusions located and identified in about $100 \mathrm{~h}$ only $(48 \mathrm{~h}$ for the experiment itself and $50 \mathrm{~h}$ for the total processing and survey of the results). Given that the experiment was not performed in the most powerful mode of the ESRF and that the motors we used were not really adapted to such kind of experiment, it would be quite easy to double the amount of amber imaged during the same time. Such a highly productive survey would be impossible with classical techniques, even on translucent amber, because it is necessary to polish it before viewing inclusions clearly. This time-consuming and potentially dangerous step (as inclusions near the surface can be lost during the polishing) is unnecessary with the protocol we present here (which could also be applied on translucent amber).

Determination of biological inclusions remains insufficient both qualitatively (the ordinal level being generally poorly indicative) and quantitatively (with $47 \%$ of undetermined animal inclusions). Increasing the resolution may help to better detect and determine small inclusions, but it must be done without loss of beam coherence and quality. Preliminary tests performed at $1.4 \mu \mathrm{m}$ pixel size with a multilayer monochromator instead of the double crystal silicon one were not effective. To keep a reasonable acquisi- tion time, it was necessary to use the multilayer monochromator, as it provides 100 times more flux than the silicon monochromator due to a larger energy bandwidth. The higher resolution reveals smaller details, but the partial loss of beam coherence and quality due to the multilayer monochromator leads to a lower overall visibility of the inclusions (Fig. 4B). Comparison between pictures obtained at $5 \mu \mathrm{m}$ and at $1.4 \mu \mathrm{m}$ demonstrates that it would be difficult to detect most of the inclusions by working with higher resolution but lower beam quality. To obtain a good visibility of the inclusion, the beam coherence has to be high enough to reveal large structures, which would not be possible with conventional sources. Despite the fact that propagation phase contrast can be used with conventional sources for microtomographic imaging at high resolution for insects in amber (Dierick et al., 2007), it would not be possible to use this approach for effective biological inclusion detection in fully opaque amber on a large scale.

Synchrotron or conventional phase contrast microtomography can bring effective results on fossil organisms in amber as soon as they are precisely characterized and located. It is not practical to apply this method to every inclusion or to use low resolution microtomography to detect inclusions in large amounts of amber. Currently, optimized propagation phase contrast X-ray microradiography appears to be the only viable solution to efficiently study fully opaque amber on a large scale, allowing us to assess fossil biodiversity and ecotype distribution based on numerous organisms determined up to the ordinal or familial level. $\mu \mathrm{CT}$ (synchrotron or conventional) remains absolutely required for more accurate determinations (e.g., Figs. 4, 5).

\section{Invisible Fossils and the Type Problem}

Phase contrast X-ray imaging techniques open new avenues of studies of opaque amber inclusions that will result in descriptions of new taxa whose reference specimen (holotype) will be invisible. It is legitimate to wonder about the accessibility of such holotypes. To solve this problem, we propose that types of new species described from fully opaque amber should include the amber sample, raw data (original microtomographic slices), segmented data (the virtually extracted organism), and 3D printouts of the organism (Fig. 5C). Having 3D printouts of the virtual types would allow direct observations without computers or microscopes (as the specimen can be printed at a larger scale than the original one), and would facilitate the diffusion of knowledge, as files for 3D printing can be easily shared. The whole process is summarized in the Figure 6.

\section{CONCLUSION}

In addition to the richness of biological inclusions in opaque amber from Charentes, this study demonstrated that propa- 

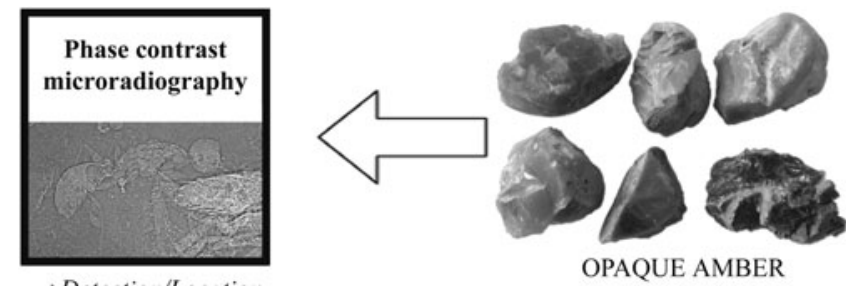

$\rightarrow$ Detection/Location
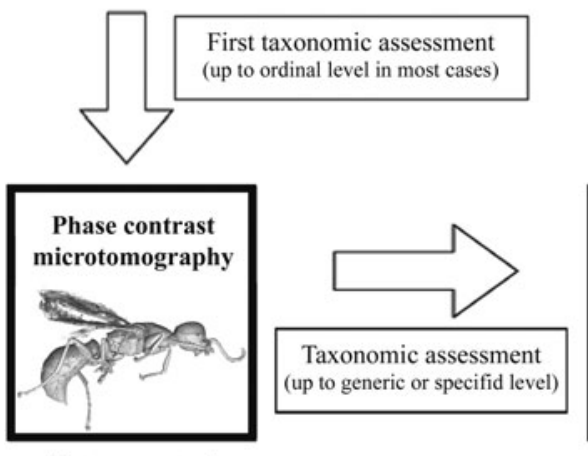

$\rightarrow$ Data segmentation

$\rightarrow$ Virtual extraction

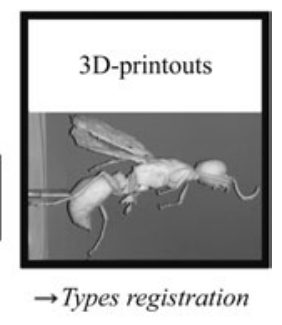

Figure 6. Diagram of the whole process from the opaque amber pieces to the final description of a specimen. Top right: Opaque amber pieces. Top left: Detection of inclusions using phase contrast radiography after immersion in water. Bottom left: Phase contrast microtomography and 3D segmentation of the specimen. Bottom right: $3 \mathrm{D}$ print of the specimen to facilitate description and for new species type registration.

gation phase constrast X-ray synchrotron imaging is an invaluable tool to gain access to opaque amber, which was not previously studied due to technical limitations. We developed an effective and rapid protocol to detect, locate, and identify biological inclusions in opaque amber. Largescale taxonomic spectra obtained this way give a broad perspective on large samples, allowing statistical approaches on the taxon diversity and the distribution of paleoecological affinities. However, because of the lack of accuracy in the organism determination, neither a precise ecological or phylogenetic study could be performed based on microradiographic imaging alone. Phase contrast X-ray microtomography (with synchrotron or conventional sources) remains necessary for precise characterization of biological inclusions; their effective detection and identification is possible only with third generation synchrotrons, as at the ESRF, because it implies high coherence properties of the X-ray beam.

\section{ACKNOWLEDGMENTS}

We warmly thank J. Baruchel, E. Boller, R. Chagnon, W. Ludwig, L. Peverini, and P. Coan from the beamlines ID19 and BM05 of the ESRF for their invaluable help. We also acknowledge Edouard-Georges Emonet for his support and his help during experiments. We acknowledge G. Raffier and M. Daronat from the Axiatec company (www.axiatec. com) and D. Laroche from the ld-proto company (www.ldproto.com) for their demonstrations of the $3 \mathrm{D}$ printer's possibilities and applications in paleontology. Finally, we are grateful to Tanya Smith for her precious help on this manuscript. This work is a contribution to the French ANR project “AMBRACE” no. BLAN07-1-184190.

\section{References}

Baruchel, J., Lodini, A., Romanzetti, S., Rustichelli, F. \& Scrivani, A. (2001). Phase-contrast imaging of thin biomaterials. Biomater 22, 1515-1520.

Beckmann, F., Bonse, U., Busch, F. \& Günnewig, O. (1997). $\mathrm{X}$-ray microtomography $(\mu \mathrm{CT})$ using phase contrast for the investigation of organic matter. J Comput Assist Tomogr 21, 539-553.

Cloetens, P., Barrett, R., Baruchel, J., Guigay, J.-P. \& SchlenkER, M. (1996a). Phase objects in synchrotron radiation hard X-ray imaging. J Phys D Appl Phys 29, 133-146.

Cloetens, P., Ludwig, W., Baruchel, J., Guigay, J.-P., PernotRejmánková, P., Salomé-Pateyron, M., Schlenker, M., Buffières, J.-Y., Maire, E. \& Peix, G. (1996b). Hard X-ray phase imaging using simple propagation of a coherent synchrotron radiation beam. J Phys D Appl Phys 32, A145-A151.

Cnudde, V., Bosselaers, J., Masschaele, B., Vlassenbroeck, J., Dierick, M., Van Hoorebeke, L. \& Jacobs, P. (2006). Species description of fossil spiders supported by non-destructive highresolution X-ray CT. Geophys Res Abs 8, 08351.

Dierick, M., Cnudde, V., Masschaele, B., Vlassenbroeck, J., VAn Hoorebeke, L. \& JACOBs, P. (2007). Micro-CT of fossils preserved in amber. Nucl Instrum Methods A 580, 641-643.

Grimaldi, D., Nguyen, T. \& Ketcham, R. (2000). Ultra-highresolution X-ray computed tomography (UHR CT) and the study of fossils in amber. In Studies on Fossils in Amber, with Particular References to the Cretaceous of New Jersey, D. Grimaldi (Ed.), pp. 77-91. Leiden, The Netherlands: Backhuys Publishers.

Henderickx, H., Cnudde, V., Masschaele, B., Dierick, M., Vlassenbroeck, J. \& Van Hoorebeke, L. (2006). Description of a new fossil Pseudogarypus (Pseudoscorpiones: Pseudogarypidae) with the use of X-ray micro CT to penetrate opaque amber. Zootaxa 1305, 41-50.

Hennig, W. (1971). Insektenfossilien aus der unteren Kreide. III. Empidiformia ("Microphorinae") aus der unteren Kreide und aus dem Baltischen Bernstein; ein Vertreter der Cyclorrhapha aus der untere Kreide. Stuttgarter Beiträge zur Naturkunde (B) 232, $1-28$.

Momose, A. (1995). Demonstration of phase-contrast X-ray computed tomography using an X-ray interferometer. Nucl Instrum Methods Phys Res A 352, 622-628.

Néraudeau, D., Allain, R., Perrichot, V., Videt, B., De Lapparent de Broin, F., Guillocheau, F., Phillippe, M., Rage, J.C. \& Vullo, R. (2003). Découverte d'un dépôt paralique à bois fossiles, ambre insectifere et restes d'Iguanodontidae (Dinosauria, Ornithopoda) dans le Cénomanien inférieur de Fouras (Charente-Maritime, Sud-Ouest de la France). C R Palevol 2, 221-230. 
Néraudeau, D., Perrichot, V., Colin, J.P., Girard, V., Gomez, B., Guillocheau, F., Masure, E., Рeyrot, D., Tostain, F., Videt, B. \& Vullo, R. (2008). A new amber deposit from the cretaceous (uppermost Albian-lowermost Cenomanian) of SW France. Cretaceous Res., in press.

Néraudeau, D., Perrichot, V., Dejax, J., Masure, E., Nel, A., Philippe, M., Moreau, P., Guillocheau, F. \& Guyot, T. (2002). Un nouveau gisement à ambre insectifère et à végétaux (Albien terminal probable): Archingeay (Charente-Maritime, France). Geobios 35, 233-240.

Perrichot, V. (2004). Early cretaceous amber from south-western France: Insight into the Mesozoic litter fauna. Geologica Acta 2, 9-22.

Perrichot, V. (2005). Environnements paraliques à ambre et à végétaux du Crétacé nord-aquitain (Charentes, Sud-Ouest de la France). Mémoires de Géosciences Rennes, 118, 310 pp.

Perrichot, V., Nel, A. \& Néraudeau, D. (2007). Schizopterid bugs (Insecta: heteroptera) in mid-Cretaceous ambers from France and Myanmar (Burma). Palaeontology 50, 1367-1374.
Polcyn, M.J., Rogers, J.V., II, Kobayashi, Y. \& Jacobs, L.L. (2003). Computed tomography of an Anolis lizard in dominican amber: Systematic, taphonomic, biogeographic, and evolutionary implications. Palaeontologia Electronica 5, 13 pp.

Snigirev, A., Snigireva, I., Kohn, V., Kuznetsov, S. \& ScheLOKOV, I. (1995). On the possibilities of X-ray phase contrast microimaging by coherent high-energy synchrotron radiation. Rev Sci Instrum 66, 5486-5492.

Tafforeau, P., Boistel, R., Boller, E., Bravin, A., Brunet, M., Chaimanee, Y., Cloetens, P., Feist, M., Hoszowska, J., Jaeger, J.-J., Kay, R.F., Lazzari, V., Marivaux, L., Nel, A., Nemoz, C., Thibault, X., Vignaud, P. \& Zabler, S. (2006). Applications of X-ray synchrotron microtomography for nondestructive 3D studies of paleontological specimens. Appl Phys A Mater Sci Process 83, 195-202.

Wilkins, S.W., Gureyev, T.E., Gao, D., Pogany, A. \& Stevenson, A.W. (1996). Phase-contrast imaging using polychromatic hard X-rays. Nature 384, 335-338. 